\title{
G

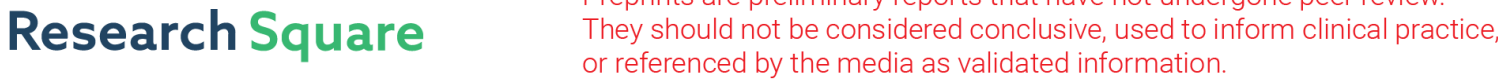 \\ Late-Onset of Chronic Granulomatous Disease Revealed By Paecilomyces Lilacinus Cutaneous Infection
}

\section{Clément Lemaigre}

University of Poitiers

\section{Felipe Suarez}

APHP- centre Université de Paris, Institut Imagine

Jean-Philippe Martellosio

University of Poitiers

Cindy Barbarin

University of Poitiers

Kevin Brunet

University of Poitiers

\section{Ewa Hainaut}

CHU Poitiers, service de dermatologie

\section{Blandine Rammaert}

University of Poitiers

France Roblot

University of Poitiers

Jose Miguel Torregrosa-Díaz ( $\nabla$ jmtorregrosad@gmail.com )

CHU Poitiers: Centre Hospitalier Universitaire de Poitiers https://orcid.org/0000-0002-5933-8562

\section{Research Article}

Keywords: Chronic granulomatous disease, clonal hematopoiesis, invasive fungal disease, antifungal prophylaxis

Posted Date: May 27th, 2021

DOI: https://doi.org/10.21203/rs.3.rs-516219/v1

License: (c) (1) This work is licensed under a Creative Commons Attribution 4.0 International License. Read Full License 
Version of Record: A version of this preprint was published at Journal of Clinical Immunology on October 1st, 2021. See the published version at https://doi.org/10.1007/s10875-021-01140-1. 


\section{Abstract}

Chronic granulomatous disease (CGD) is an inherited immunodeficiency due to defective leukocyte NADPH responsible for recurrent infections and aberrant inflammation. Mutations in the $C Y B B$ gene are responsible for the X-linked CGD and account for approximately $70 \%$ of the cases. CGD is diagnosed during childhood in males. Female carriers may have biased $\mathrm{X}$ inactivation and may present with clinical manifestations depending on the level of residual NADPH oxidase activity. We report the case of a previously asymptomatic female carrier who was diagnosed at age 67 with a skin infection with the rare fungus, Paecylomyces lilacinus as the first manifestation of CGD. Dihydrorhodamin 123 (DHR) activity was below 10\%. Next-generation sequencing (NGS) revealed mutations in DNMT3A, ASXL1, and STAG2 suggesting that clonal hematopoiesis of undetermined potential (CHIP) could be responsible for a progressive loss of NADPH oxidase activity and the late onset of X-linked CGD in this patient. Long-term follow-up of asymptomatic carrier women seems to be essential after 50 years old.

\section{Introduction}

Chronic granulomatous disease (CGD) is a primary immune deficiency associated with reduced NADPH oxidase activity in phagocytes. NADPH oxidase complex is responsible for the production of free radicals leading to oxidative burst and microorganism killing. The different forms of CGD are associated with inactivating mutations in genes coding for the subunits of the phagocyte NADPH oxidase complex. Subunits Gp91, encoded by CYBB gene (X-linked), and p22 encoded by the CYBA gene (autosomal recessive) are located on the lysosomal membrane. The other subunits encoded by the NCF 1-4 genes (subunit p22, p47, p67, and p40phox) are localized on the cytosolic side of the lysosomal membrane and regulate the catalytic activity of complex [1-3].

The X-linked form is the most common form, usually diagnosed around five years old, rarely after 20 [4]. The prognosis is poor due to severe recurrent infections and granulomatous inflammation affecting various organs. Despite antibacterial and antifungal prophylaxis, the mean survival time is 37.8 years in the X-linked form, and 49.6 years in the autosomal recessive form [4]. Although X-linked form preferentially affects men, $8 \%$ of cases occur in women due to unfavorable lyonization or new mutation of the active $\mathrm{X}$ chromosome $[4,5]$.

Today, CGD is diagnosed mostly by quantifying the production of hydrogen peroxide $\left(\mathrm{H}_{2} \mathrm{O}_{2}\right)$ by dihydrorhodamine oxidation (DHR). Susceptibility to infections is apparent in female X-inked CGD carriers with DHR below $20 \%$ (and especially below 10\%) in particular with opportunistic pathogens such as Nocardia spp., Bukholderia spp., and invasive fungi such as Aspergillus spp [1-3]. Autoimmune or inflammatory manifestations of the disease such as discoid lupus erythematosus, Raynaud's phenomenon, and granulomatous colitis may be present regardless of the DHR level [5].

We report the case of a female carrier who developed a symptomatic form of X-linked CGD with DHR < $10 \%$ at the age of 67 years old and hypothesized that the development of clonal hematopoiesis could be 
responsible for the late-onset disease.

\section{Case Description}

The patient was a 67-year-old woman, known to be an asymptomatic carrier of X-linked CGD (Gp91 subunit mutation) following the death of one of her children due to salmonellosis in 1985. She had two other sons X-linked CGD, one who died in 1988 at the age of 7 from invasive aspergillosis, and one with a history of recurrent bacterial and fungal infections (aspergillosis) and inflammatory bowel disease, alive and well at age of 35 . The patient had never manifested with infections or inflammatory symptoms related to CGD until she was referred to our unit. She also had a history of breast cancer treated by tumorectomy, hormonal therapy, and radiotherapy at age 62 years in 2013.

The patient presented with skin lesions of her right thumb in December 2018. She had worked as a sheep farmer for many years. The papules evolved towards pustules with purulent discharge on an inflammatory background since December 2018 despite two different lines of oral antibiotic therapy and valaciclovir. Biopsies were performed in mars 2019 after she was referred to our department (Figure 1). All biopsy cultures grew with a mold confirmed by sequencing as Paecylomyces lilacinus. Histopathological examination showed an inflammatory reaction and mycelial elements compatible with invasive fungal disease. Other microbiological investigations remained negative, especially poxvirus PCR. The patient was treated with oral voriconazole, loading dose $400 \mathrm{mg}$ BID then $200 \mathrm{mg}$ BID for 3 months. Treatment was well tolerated and allowed complete healing of the lesions. Her family history and the infection with a rare fungus led us to explore the possibility of immune deficiency linked to CGD. DHR was found to be $<10 \%$ consistent with a skewed X-inactivation. As the patient had no infectious or inflammatory manifestations of X-linked CGD before age 67, we hypothesized that the severely decreased DHR was the consequence of an acquired skewing of X-chromosome. Next-generation sequencing (NGS) was performed in mononucleated cells of the patient's bone marrow. We found variants in DNMT3A with a variant allele frequency (VAF) of 7,88 \%, ASXL1 (VAF 47,94\%), and STAG2 (VAF 48,26\%). The patient had normal blood counts and no signs of myelodysplasia were noted on the bone marrow aspirate, consistent with the presence of clonal hematopoiesis of indeterminate potential. Prolonged secondary prophylaxis with oral posaconazole and cotrimoxazole was indicated to prevent bacterial and fungal infections in this patient with late-onset severe CGD.

\section{Discussion}

This case addresses the issue of a late onset of CGD manifested by an invasive fungal infection due to a rare environmental mold, $P$. liliacinus at age 67 in a previously asymptomatic female carrier of $X$-linked CGD (Gp91 subunit mutation, exon 7, c.676C>T, p.Arg226*).

Three other cases of late-onset CGD have been described in the literature, all three patients being women aged 45,53 , and 64 [6-8]. These cases were diagnosed after infection by opportunistic pathogens (Aspergillus spp [6], pulmonary infection with Burkholderia cepacian [7], and Serratia marcescens [8]). 
Invasive fungal diseases due to molds other than Aspergillus spp. is rare during CGD course [9]. $P$. lilacinus is a cosmopolitan saprophytic soil and plant filamentous fungus, with a particular tropism for eyes, skin, and sinuses. Skin is the second most common site of infection after ophthalmic involvement with various unspecific manifestations of the disease from erythematous papules to necrotic lesions or cellulitis [10].

Age-related clonal hematopoiesis is common, found in roughly $2-3 \%$ of adults over the age of 50 . It is associated with the risk of subsequent hematological malignancies and atherosclerosis and could be the key factor to understand the late onset of CGD in our patient. Clonal hematopoiesis is a frequent phenomenon correlated with medullary aging [11-13], so 2-3\% of the population over 50 years old might be concerned [14]. It consists of the emerging of a related cell clone with an acquired gene mutation. The selection of one or more stem cells having a proliferation advantage can gradually replace the other stem cells in some cases [12], leading to reduced genetic diversity of hematopoiesis via the selection of one or more stem cells to the detriment of others. Two major consequences are known to date, increased cardiovascular risk and a variable rate of myelodysplasia and acute leukemia $[15,16]$. Wolach and al. have described for the first time a late onset of X-linked CGD in a 64-year-old woman and have suspected this phenomenon without being able to prove it [8]. In our patient, two of the three mutations most frequently involved in clonal hematopoiesis (DNMT3A and $A S X L 1$ ) [17] were found. DNMT3A gene is located on chromosome 2 and encodes DNA (cytosine-5)-methyltransferase $3 \mathrm{~A}$, an epigenetic modifier essential in hematopoietic stem cell differentiation [18]. It may confer a proliferation advantage becoming more important within stem cells aging [19]. ASXL1 gene is an epigenetic marker frequently involved in all subtypes of myeloid malignancies and also in clonal hematopoiesis [20].

X-inactivation is an embryological phenomenon that is permanent at each cell division but whose allelic ratio varies throughout life [21]. Although the depth of the deficit, an atypical infection was not detected in our patient until the age of 67 years old. Hereby, we hypothesize that it is unlikely that our patient has had a so deep impaired DHR since birth. The skewed X-inactivation in favor of the mutated CYBB gene could have led to the progressive appearance of the genotype and phenotype disease. This asymmetry could be explained by the age-related selection of a cell carrying the mutation [20], as in our patient we found probable clonal hematopoiesis, so a plausible explanation could be that if the predominant clone carries the $C Y B B$ mutation, the pattern of inactivation of $\mathrm{X}$ becomes asymmetric in favor of this mutation. That would have led to the progressive reduction of the DHR and thus the acquisition of the disease. Interestingly, the patient had been treated by radiotherapy 5 years before, so the acquisition of clonal hematopoiesis could have been accelerated by this treatment.

Our finding suggests that asymptomatic carriers of $C Y B B$ mutations should have life-long clinical and biological surveillance. Yearly clinical exam and DHR measurement should start at 50 years old when the risk of clonal hematopoiesis becomes significant, or before if the patient starts to have inflammatory symptoms. Inflammatory symptoms like lupus discoid, pulmonary fibrosis, Crohn-like disease, can appear before infection when DHR is $>20 \%$. Antifungal and antibacterial prophylaxis should be started as soon as the DHR is $<20 \%$, a percentage below which the risk of infection prevails [5]. 
In conclusion, the X-linked form of CGD could affect older women due to clonal hematopoiesis. Close monitoring of women carrying the mutation by studying phagocytic function should be interesting to detect DHR decrease below $20 \%$. This strategy could help to prevent infections by starting early chemoprophylaxis and environmental prevention measures.

\section{Declarations}

Funding: no funding was obtained to support this work.

Conflicts of interest/Competing interests. None of the authors declare any conflict of interest.

Consent to participate. the patient consents to the use of her clinical information to the redaction of this case report.

Consent for publication. the patient consents to the use of her clinical information to be published for this case report.

\section{References}

1. Kuhns DB, Alvord WG, Heller T, Feld JJ, Pike KM, Marciano BE, et al. Residual NADPH Oxidase and Survival in Chronic Granulomatous Disease. N Engl J Med. 2010;363:2600-10.

2. Winkelstein JA, Marino MC, Johnston RB, Boyle J, Curnutte J, Gallin JI, et al. Chronic Granulomatous Disease: Report on a National Registry of 368 Patients: Medicine. 2000;79:155-69.

3. Holland SM. Chronic Granulomatous Disease. Hematology/Oncology Clinics of North America. 2013;27:89-99.

4. van den Berg JM, van Koppen E, Åhlin A, Belohradsky BH, Bernatowska E, Corbeel L, et al. Chronic Granulomatous Disease: The European Experience. Alspaugh A, editor. PLoS ONE. 2009;4:e5234.

5. Marciano BE, Zerbe CS, Falcone EL, Ding L, DeRavin SS, Daub J, et al. X-linked carriers of chronic granulomatous disease: Illness, lyonization, and stability. Journal of Allergy and Clinical Immunology. 2018;141:365-71.

6. Rösen-Wolff A, Soldan W, Heyne K, Bickhardt J, Gahr M, Roesler J. Increased susceptibility of a carrier of X-linked chronic granulomatous disease (CGD) to Aspergillus fumigatus infection associated with age-related skewing of lyonization. Annals of Hematology. 2001;80:113-5.

7. Sarwar G, Malmanche T, Rassam L, Grainge C, Williams A, Arnold D. Chronic granulomatous disease presenting as refractory pneumonia in late adulthood. Respirology Case Reports. 2015;3:54-6.

8. Wolach B, Scharf Y, Gavrieli R, de Boer M, Roos D. Unusual late presentation of X-linked chronic granulomatous disease in an adult female with a somatic mosaic for a novel mutation in CYBB. Blood. 2005;105:61-6.

9. Henriet S, Verweij PE, Holland SM, Warris A. Invasive Fungal Infections in Patients with Chronic Granulomatous Disease. In: Curtis N, Finn A, Pollard AJ, editors. Hot Topics in Infection and 
Immunity in Children IX [Internet]. New York, NY: Springer New York; 2013 [cited 2021 May 6]. p. 2755. Available from: http://link.springer.com/10.1007/978-1-4614-4726-9_3

10. Pastor FJ, Guarro J. Clinical manifestations, treatment and outcome of Paecilomyces lilacinus infections. Clinical Microbiology and Infection. 2006;12:948-60.

11. Shlush LI. Age-related clonal hematopoiesis. Blood. 2018;131:496-504.

12. Groarke EM, Young NS. Aging and Hematopoiesis. Clinics in Geriatric Medicine. 2019;35:285-93.

13. Zink F, Stacey SN, Norddahl GL, Frigge ML, Magnusson OT, Jonsdottir I, et al. Clonal hematopoiesis, with and without candidate driver mutations, is common in the elderly. Blood. 2017;130:742-52.

14. Steensma DP. Clinical consequences of clonal hematopoiesis of indeterminate potential. Blood Advances. 2018;2:3404-10.

15. Sano S, Wang Y, Walsh K. Clonal Hematopoiesis and Its Impact on Cardiovascular Disease. Circ J. 2018;83:2-11.

16. Desai P, Hassane D, Roboz GJ. Clonal Hematopoiesis and risk of Acute Myeloid Leukemia. Best Practice \& Research Clinical Haematology. 2019;32:177-85.

17. Jaiswal S, Fontanillas P, Flannick J, Manning A, Grauman PV, Mar BG, et al. Age-Related Clonal Hematopoiesis Associated with Adverse Outcomes. N Engl J Med. 2014;371:2488-98.

18. Challen GA, Sun D, Jeong M, Luo M, Jelinek J, Berg JS, et al. Dnmt3a is essential for hematopoietic stem cell differentiation. Nat Genet. 2012;44:23-31.

19. Buscarlet M, Provost S, Zada YF, Barhdadi A, Bourgoin V, Lépine G, et al. DNMT3A and TET2 dominate clonal hematopoiesis and demonstrate benign phenotypes and different genetic predispositions. Blood. 2017;130:753-62.

20. Busque L, Mio R, Mattioli J, Brais E, Blais N, Lalonde Y, et al. Nonrandom X-inactivation patterns in normal females: Iyonization ratios vary with age. Blood. 1996;88:59-65.

21. Mengel-From J, Lindahl-Jacobsen R, Nygaard M, Soerensen M, Ørstavik KH, Hertz JM, et al. Skewness of $\mathrm{X}$-chromosome inactivation increases with age and varies across birth cohorts in elderly Danish women. Sci Rep. 2021;11:4326.

\section{Figures}



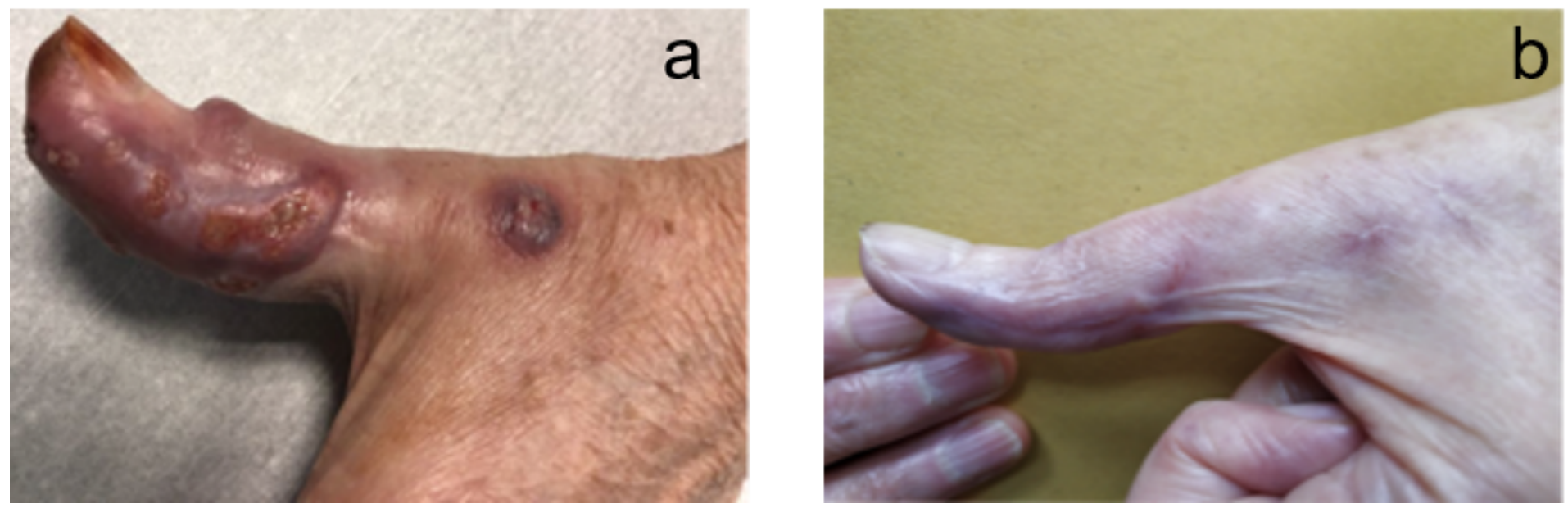

Figure 1

a. Right thumb before treatment $b$. Right thumb after treatment 